\title{
A multi-generation risk assessment of Cry1F on the non-target soil organism Folsomia candida (Collembola) based on whole transcriptome profiling
}

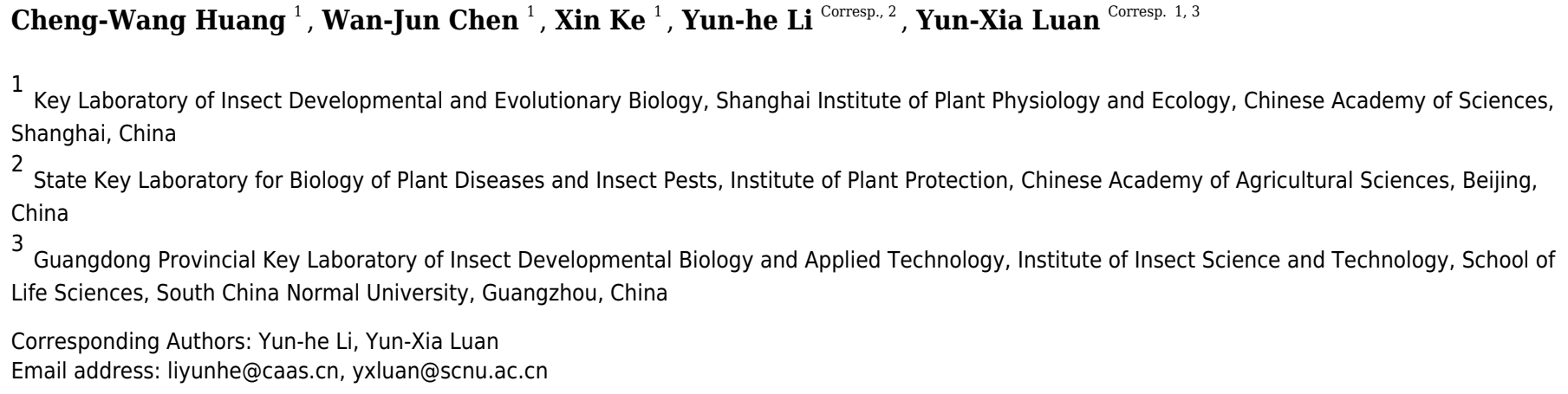

The Bacillus thuringiensis (Bt) toxin Cry1F has been used to develop insect-resistant genetically engineered (IRGE) crops. There has been great interest in evaluating its potential risk to non-target organisms (NTOs). However, the majority of previous risk assessments only examined one generation of NTOs using several physiological indicators, which cannot comprehensively detect some potential sub-lethal effects at the molecular level. In this study, we conducted a laboratory-based, multi-generational risk assessment of CrylF for the collembolan Folsomia candida, an important representative of soil arthropods in terms of survival, reproduction, and differentially expressed genes (DEGs) identified from whole transcriptome profiles. Our results demonstrated that Cry1F was continuously ingested by collembolans over three consecutive generations, but it did not affect the survival or reproduction of $F$. candida. There were no significant differences in the global gene expression between $F$. candida-fed diets with and without Cry1F, and no consistent co-expressed DEGs over three generations. In addition, Cry1F did not obviously alter the expression profiles of seven sensitive biological markers. Our composite data indicates that CrylF had no long-term harmful effects on collembolan F. candida. 
1 A multi-generational risk assessment of Cry1F on the non-target soil organism Folsomia candida (Collembola) based on whole transcriptome profiling

$6 \quad{ }^{1}$ Key Laboratory of Insect Developmental and Evolutionary Biology, Shanghai Institute of Plant

7 Physiology and Ecology, Chinese Academy of Sciences, Shanghai, China

$8 \quad{ }^{2}$ State Key Laboratory for Biology of Plant Diseases and Insect Pests, Institute of Plant

9 Protection, Chinese Academy of Agricultural Sciences, Beijing, China

$10 \quad{ }^{3}$ Guangdong Provincial Key Laboratory of Insect Developmental Biology and Applied

11 Technology, Institute of Insect Science and Technology, School of Life Sciences, South China

12 Normal University, Guangzhou, China.

14 Corresponding Authors:

Yun-Xia Luan ${ }^{1,3}$ and Yun-he $\mathrm{Li}^{2}$

Email address: yxluan@scnu.ac.cn; liyunhe@,caas.cn 


\section{Abstract}

18 The Bacillus thuringiensis (Bt) toxin Cry1F has been used to develop insect-resistant genetically 19 engineered (IRGE) crops. There has been great interest in evaluating its potential risk to nontarget organisms (NTOs). However, the majority of previous risk assessments only examined one generation of NTOs using several physiological indicators, which cannot comprehensively detect some potential sub-lethal effects at the molecular level. In this study, we conducted a laboratorybased, multi-generational risk assessment of Cry1F for the collembolan Folsomia candida, an important representative of soil arthropods in terms of survival, reproduction, and differentially expressed genes (DEGs) identified from whole transcriptome profiles. Our results demonstrated that Cry1F was continuously ingested by collembolans over three consecutive generations, but it did not affect the survival or reproduction of $F$. candida. There were no significant differences in the global gene expression between $F$. candida-fed diets with and without Cry $1 \mathrm{~F}$, and no consistent co-expressed DEGs over three generations. In addition, Cry1F did not obviously alter the expression profiles of seven sensitive biological markers. Our composite data indicates that Cry1F had no long-term harmful effects on collembolan $F$. candida. 


\section{Introduction}

Over the past 20 years, genetically engineered (GE) crops have been widely planted throughout the world. The most common of these are insect-resistant genetically engineered (IRGE) crops, including IRGE cotton, maize, and others (ISAAA, 2016). All the current commercialized IRGE crops express cry or vip genes derived from the bacterium Bacillus thuringiensis $(B t)$, and these genes encode insecticidal proteins targeting lepidopteran or coleopteran insect pests (Bravo et al., 2011; Liu et al., 2016; Zhao et al., 2016). Although the planting of IRGE crops will greatly reduce the use of broad-spectrum insecticides, the potential risks of IRGE crops to the environment and human health must be assessed prior to their commercialization. One of the assessments is their potential negative effects on non-target organisms (NTOs) within agroecosystems based on a tiered approach from laboratory to field (EFSA, 2010; Li et al., 2014; Romeis et al., 2008; Shelton, Zhao \& Roush, 2002; Svobodová, 2015). To date, substantial laboratory and field tests have been conducted with many different NTOs belonging to a range of orders, e.g. ladybird beetles, honeybees, parasitic wasps, collembolans, earthworm, and zebrafish, covering different functional groups, including herbivores, predators, parasitoids, and detritivores (Gao et al., 2018; ILSI Research Foundation, 2013; Jia et al., 2016; Marques et al., 2018; Neher et al., 2014; Tian et al, 2014; Svobodová et al., 2015).

Collembolans are an important group of detritivore soil arthropods because of their large numbers in the soil $\left(10^{4}-10^{5} \mathrm{~m}^{-2}\right)$, great contribution to the decomposition of plant residues, and sensitivity to many soil pollutants (Buch et al., 2016; Chen et al., 2015; Hopkin, 1997; Zortéa et $a l ., 2015)$. They are commonly exposed to $B t$ toxins when IRGE crop residues decompose in agricultural lands (Li et al., 2007; Valldor et al., 2015). As a standard test organism that is often used for toxicological, biosafety, and environmental assessment, the parthenogenetic collembolan $F$. candida is also an important NTO for the risk assessment of IRGE crops. Most previous risk assessments found no harmful effects of various $B t$ toxins on collembolans, regardless of whether the testing was done in the laboratory or field (ILSI Research Foundation, 2013; Marques et al., 2018; Sims \& Martin, 1997; Yang et al., 2015, 2018; Zhang et al., 2017a). However, most previous risk assessments have been limited to several physiological indicators, e.g. survival, reproduction, and some enzyme activities, which cannot detect potential weak effects. In addition, most studies only examined one generation of $F$. candida, i.e., they did not evaluate the potential risk to the collembolan progeny.

As the field case always involves "multi-generational" exposure, some potential sub-lethal effects of an environmental factor may cumulate over time and become apparent only in a living organism after exposure over multiple generations. Noordhoek et al. (2018) found a metal-based nanomaterial tungsten carbide-cobalt affected the reproduction and survival of $F$. candida from the third generation. The current one-generation laboratory risk assessment cannot comprehensively evaluate the potential nonlethal chronic effects of $B t$ toxins on NTOs. By using 
multi-generational laboratory risk assessments, Bakonyi et al. (2011) and Szabó, Seres \& Bakonyi (2017) found that $F$. candida that were fed CrylAb expressing maize leaves had some alterations in life-history traits and reproduction. To our knowledge, these are the only studies that have evaluated the long-term potential risk of $B t$ toxins to collembolans. However, it is difficult to tell whether the effects were caused by the $B t$ toxin or other components of the maize leaves and additional studies are needed.

In assessing the effects of $B t$ toxins on collembolans, most previous studies have used a finite number of physiological indices (survival rates, reproductive rates, developmental duration, etc.) or biological markers (enzyme activity, midgut bacterial diversity, etc.). These traditional methods of risk assessment can reflect the life status of collembolans, but may not detect slight changes at the molecular level. By using microarray and qPCR, Yuan et al. (2014) found eleven gene transcripts of $F$. candida that responded to the $B t$ proteins (Cry1Ab and Cry1Ac). RNA sequencing (RNA-Seq), which is different from microarray and qPCR, can provide a whole transcriptome profile of NTOs to check stress-responsive changes in the gene expression, which makes it possible to evaluate unknown effects, and helps us find the potential non-lethal chronic toxicity of Bt. Based on RNA-Seq data, Zhang et al. (2017b) annotated 32,268 unigenes of the Chinese green mussel (Perna viridis) and identified 9,048 differentially expressed genes (DEGs) between exposed and non-exposed groups to cadmium, suggesting a sensitive response of the mussel transcriptome to cadmium. Noordhoek et al. (2018) studied phenotypic effects and transcriptional responses in $F$. candida exposed to nanomaterials and detected physiological alterations cumulating from one generation to the next based on gene expression assays but without visible phenotypic variables.

Cry $1 \mathrm{~F}$ has been transferred to maize, cotton, and others, and previous studies have found no harmful effects of Cry $1 \mathrm{~F}$ on some tested NTOs, including F. candida (Higgins et al., 2009; ILSI Research Foundation, 2013; Kim et al., 2012; Marques et al., 2018; Tian et al., 2014). However, its risk to collembolans has not been evaluated as comprehensively as other Cry proteins such as Cry1 Ab, Cry1 Ac, Cry2A, and Cry1C. In this study, we performed a dietary exposure experiment (DEE) with $F$. candida that was fed an artificial diet containing or excluding purified Cry $1 \mathrm{~F}$ protein for three consecutive generations. We compared the effects of these two diets on the survival, reproduction, and transcriptome changes of $F$. candida for each generation. To our knowledge, this is the first multi-generational laboratory risk assessment of Cry $1 \mathrm{~F}$ on $F$. candida, and also the first attempt to use RNA-Seq to evaluate the impact of a $B t$ toxin on the gene expression of $F$. candida.

\section{Materials and methods}

\section{Test organism.}

The Danish strain of the parthenogenetic Folsomia candida Willem, 1902, was originally obtained from Aarhus University, Denmark, and has been cultured in our laboratory for over 10 
114 years. F. candida is usually fed granulated dried baker's yeast (Hebei Mauri Foods Co., Ltd., 115 Zhangjiakou, China) and was reared in the Petri dishes (90 mm x $18 \mathrm{~mm}$ ) containing a 5-7 mm 116 layer of a solidified mixture of plaster of Paris and activated charcoal (9:1 wt/wt, dissolved in 117 distilled water, with about $270 \mathrm{ml}$ of water $/ 500 \mathrm{~g}$ of mixed powder) (Fig. 1A-C). The baker's 118 yeast was placed on the surface of the plaster and was renewed weekly to reduce the growth of 119 other fungi. Distilled water was added to the base as needed such that free water was present in 120 the plaster pores but did not form a film on the plaster surface. These dishes with $F$. candida

121

122

123

124

125

126

127

128

129

130

131

132

133

134

135

136

137

138

139

140

141

142

143

144

145

146

147

148

149

150

151

152

153

154

\section{Dietary exposure experiment (DEE).}

The artificial diet containing Cry1F (BT diet) was prepared as follows: $2 \mathrm{mg}$ of purified Cry1F protein (Envirologix Inc., Portland, Maine, USA) and $4 \mathrm{~g}$ of baker's yeast granules were dissolved in $10 \mathrm{ml}$ of distilled water; the preparation was fully mixed and then transferred into a plastic container. The final concentration of Cry1F protein in the BT diet was $500 \mu \mathrm{g} / \mathrm{g}$, which is much higher than the highest Cry1F protein concentrations $(38.4 \mu \mathrm{g} / \mathrm{g})$ estimated in IRGE crops (USDA, 2003,2004 ), as well as than the $\mathrm{EC}_{50}$ values of Cry1F against lepidopteran pests Chilo suppressalis $(6 \mu \mathrm{g} / \mathrm{g})$ and larvae of Bombyx mori (136 ng/g) (Jiao et al., 2016). After $27 \mathrm{~h}$ of lyophilization, the BT diet was ground into powder and stored at $-70^{\circ} \mathrm{C}$. As the control, baker's yeast granules without Cry1F (CT diet) were prepared in the same way. We also tried a positive control by adding the insecticidal compounds (potassium arsenate) into the baker's yeast granules, but it significantly affected the survival and the development of $F$. candida, which hampered the performance of the consecutive multi-generational exposure experiment. Therefore, we didn't perform the positive control in our multi-generational DEE. However, the concentration of Cry1F in the BT diet was high enough to validate the test of Cry1F to F. candida.

Based on the Organization for Economic Cooperation and Development (OECD) test protocol (OECD, 2016), we designed a multi-generational DEE on F. candida (Fig. 1D). During the experiment, all $F$. candida were cultured in the same artificial climate chamber with the culture conditions mentioned above and were synchronized to the same stage (neonates) at the start of each generation in the experiment.

First, we transferred 80-100 randomly selected $F$. candida adults (about 4-6 weeks old) to a new Petri dish. After $72 \mathrm{~h}$ of oviposition, all adults were removed. The eggs began to hatch 10 days later. After $24 \mathrm{~h}$ of hatching, we selected synchronized neonates. They were transferred to a new Petri dish and were fed with pure baker's yeast for 10 days. Next, all juveniles were separated into two sets: the BT set were fed BT diet, and the CT set were fed CT diet. Each set was done with individually- and group-reared collembolans.

In the first-generation DEE, 60 juveniles were transferred to 60 small plastic Petri dishes (55 $\mathrm{mm}$ x $14 \mathrm{~mm}$, plaster height: $5 \mathrm{~mm}$ ), respectively, as individually-reared collembolans. 30 were fed the BT diet, and 30 were fed the CT diet. The remaining juveniles were evenly distributed 
155

156

157

158

159

160

161

162

163

164

165

166

167

into six plastic Petri dishes ( $85 \mathrm{~mm}$ x $16 \mathrm{~mm}$, plaster height: $5 \mathrm{~mm}$ ), with 60 to 80 collembolans per dish. Three of them were fed the BT diet and the other three were fed the CT diet. All diets were renewed every 2 days to avoid Cry $1 \mathrm{~F}$ protein degradation and the growth of other fungi.

The first generation was assessed after 25 days of DEE, which is 35 days from hatching. We choose this time point to ensure that $F$. candida had sufficient exposure to Cry1F, and had laid 12 batches of eggs, but with only one batch of eggs hatching. For the individually-reared collembolans, the numbers of adults and juveniles in each treatment were counted to calculate the survival and reproduction rates. For the collembolans fed in groups, about 20 individuals per replicate were randomly collected for ELISA (enzyme-linked immunosorbent assay, see section ELISA below), and 25-35 individuals per replicate were collected for RNA-Seq (see section $R N A$ extraction, sequencing and unigene annotation below).

The remaining collembolans fed in groups of CT and BT sets were used for synchronization of the second generation. To maintain the same experimental conditions, the CT and BT sets were synchronized independently, and their neonates were fed the CT diet or BT diet directly after birth. After 10 days, the second-generation DEE began and was conducted in the same manner as the first-generation DEE except that there were 40 replicates per set for individually-reared collembolans and two replicates per set for group-reared collembolans. After 25 days of DEE, survival and reproduction were evaluated using the individually-reared collembolans. ELISA and RNA-Seq were performed using the group-reared collembolans as described for the first generation. The remaining collembolans that were fed in groups in the second generation were used to start the third generation. The procedure for the third-generation DEE was identical to that for the second except that there were three rather than two replicates per set for the groupreared collembolans.

\section{Survival and reproduction.}

The individually reared collembolans were used to evaluate the survival and reproduction of F. candida at the end of each generation. Several replicates were excluded from the analysis: 1 ) missing adult collembolans (5 in BT treatment and 5 in CT treatment over three generations), which are assumed to have escaped because there was no corpse left; 2) eggs didn't hatch due to overgrown fungi (6 in BT treatment and 5 in CT treatment over three generations).

With the aid of a stereoscopic microscope (Nikon, SMZ-10), the number of living adults was determined as a measure of survival, and the number of juveniles produced was determined as a measure of reproduction. We calculated the mean number of juveniles produced per individually fed collembolan. Due to the wide variation in the reproduction data (7-41 juveniles per individually-reared collembolan), the data were log-transformed prior to conducting statistical analysis. Student's $t$-test was used to analyze the differences between the two treatments in each generation. 
In addition, the data for the reproduction of all BT and CT sets over three generations were subjected to a two-way ANOVA, followed by the LSD (least significant difference) test, with diet treatment, "generation", and their interaction as fixed factors. Generation is not an independent variable, but we used "generation" as a label representing unknown variable factors in order to test whether the reproductions of $F$. candida over three generations in the same treatment were affected by unpredictable environment stress. All of these statistical analyses were performed with IBM SPSS Statistics 24 (version R24.0.0.0). Differences were considered significant at $p<0.05$.

\section{ELISA.}

The concentration of Cry $1 \mathrm{~F}$ in the diets and in the $F$. candida fed in groups was measured by ELISA for each replicate in each generation. The QuantiPlate Kit for Cry1F (Envirologix Inc., Portland, Maine, USA) was used to detect Cry1F in a 2- to 4-mg sample of the fresh diet in each replicate, in a 2- to 4-mg sample of the diet in each replicate after 2 days of feeding, and in 20 individuals of $F$. candida $(2-5 \mathrm{mg})$ per replicate. Before the test, all $F$. candida samples were washed in a PBST extraction buffer (phosphate-buffered saline with Tween-20, pH 7.4) to remove any Cry $1 \mathrm{~F}$ toxin from the outer surface of the collembolan cuticles. Then, all diet samples and $F$. candida samples were fully ground with an electric grinding rod and extracted with a PBST extraction buffer (phosphate-buffered saline with Tween-20, pH 7.4), respectively. ELISA was performed according to the manufacturer's instructions. A two-way ANOVA was used to compare Cry1F concentrations of three BT sets over three generations by using the software IBM SPSS Statistics 24 (version R24.0.0.0). Differences were considered significant at $p<0.05$.

\section{RNA extraction, sequencing, and unigene annotation.}

The miRNeasy ${ }^{\circledR}$ Mini Kit (Qiagen Biotech, Germany) was used to extract the total RNA from 25-35 individuals per replicate of the collembolans that were fed in groups for each generation. The RNA concentration and integrity was evaluated with Aglient 2100 Bioanalyzer (Agilent, USA).

The cDNA library construction, Illumina sequencing, and de novo assembly of RNA samples were carried out by Hangzhou 1gene Technology Co., Ltd. All cDNA libraries were constructed using the NEBNext ${ }^{\circledR}$ Ultra $^{\text {TM }}$ RNA Library Prep Kit for Illumina ${ }^{\circledR}$ (NEB, USA) following the protocol described by the manufacturer. The libraries were sequenced with 150-bp paired-end reads on an Illumina Hiseq 4000 platform (San Diego, CA, USA). After sequencing, the raw reads (about $6 \mathrm{~GB}$ of data for each replicate) were filtered to remove adaptor sequences, duplication sequences, and low-quality sequences. The clean reads were de novo assembled into unigenes using Trinity and SOAPdenovo-Trans. Six public databases were used to annotate unigenes with BLASTx or BLASTn (E-value $<10^{-5}$ ), including NCBI Nr and Nt databases (http://www.ncbi.nlm.nih.gov/), SwissProt (http://www.expasy.ch/sprot/), KEGG (http://www.genome.jp/kegg/), COG (http://www.ncbi.nlm.nih.gov/COG/), and GO 
237

238

239

240

241

242

243

244

245

246

247

248

249

250

251

252

253

254

255

256

257

258

259

260

261

262

263

264

265

266

267

268

269

270

271

272

273

274

275

276

(http://www.geneontology.org/).

\section{Differential gene expression and biological marker expression.}

Based on strict synchronization and multiple repeats of samples, we screened DEGs between groups of CT and BT sets in the same generation. The expression levels of all unigenes were calculated using the FPKM (fragments per kb per million fragments) method: FPKM = $\left(10^{6} \mathrm{C}\right) /\left(\mathrm{NL} / 10^{3}\right)$. The data of two or three replicates for each set in every generation were averaged, and the whole transcriptome gene expression of all CT and BT sets were compared. DEGs were determined by the condition of FC (fold change, $\mid \log 2$ ratio| of FPKM (BT vs. CT)) $\geq 1$ and FDR (false discovery rate) $\leq 0.05$. Co-expressed DEGs over three generations were screened and displayed with Venn diagrams. All DEGs over three generations were analyzed by heatmaps and the hierarchical clustering.

Additionally, we carefully checked the expression of seven known biological markers, whose changes in expression level are often used to indicate that the test organism is experiencing stress. These genes encode the following proteins: the antioxidant-related enzymes catalase (CAT) (Niu et al., 2017; Yousef, Abdelfattah \& Augustyniak, 2017) and superoxide dismutase (SOD) (Yang et al., 2015; Yousef, Abdelfattah \& Augustyniak, 2017), the detoxification-related enzymes glutathione S-transferase (GST) (Niu et al., 2017; Oliveira et al., 2015), carboxylesterase (CES) (Niu et al., 2017; Yang et al., 2015), and glutathione reductase (GR) (Yang et al., 2015), a metallothionein-like motif containing protein (MTC) (Nakamori \& Kaneko, 2013), which is unique in F. candida and which is very sensitive to different heavy metal ions, and the heat shock protein 70 (HSP70) (Liu et al., 2010), which is sensitive to insecticides, drought, and other environmental stresses. The expression profiles of unigenes annotated to these biological markers were examined and their expression levels were compared among different samples. All these genes of the seven biological markers over three generations were analyzed by heatmaps and the hierarchical clustering.

The software R (version 3.4.0) was used for the heatmaps (R package 'pheatmap' (https://cran.r-project.org/web/packages/pheatmap/index.html)) and the hierarchical clustering ('dist' and 'hclust' orders, distance method as 'euclidean', and cluster method as 'complete') of all DEGs and biological markers over three generations (expression data of DEGs were transformed by using the common logarithm of the counts plus 1).

\section{Results}

\section{Cry1F concentrations in the diet and $F$. candida.}

ELISA measures showed that no CrylF toxin was detected in the pure yeast diet or in the $F$. candida that were fed the $\mathrm{CT}$ diet. The average concentration (mean $\pm \mathrm{SE}$ ) of Cry1F was $415 \pm$ $15 \mu \mathrm{g} / \mathrm{g}$ in the fresh BT diet and $358 \pm 57 \mu \mathrm{g} / \mathrm{g}$ in the BT diet after 2 days of feeding, indicating that Cry1F was continually present in the experiment. The average concentrations of Cry $1 \mathrm{~F}$ in 
collembolans that were fed the BT diet were $3.5 \pm 1.3,2.7 \pm 1.4$, and $5.2 \pm 1.0 \mu \mathrm{g} / \mathrm{g}$ at the end of the first, second, and third generation, respectively. These concentrations were not significantly different based on a two-way ANOVA $(p=0.429)$ and suggest that the Cry1F was continuously ingested by collembolans.

\section{Survival and reproduction of $\boldsymbol{F}$. candida.}

All collembolans in both the CT and BT sets survived and reached the adult stage for all three generations, suggesting that Cry $1 \mathrm{~F}$ has no obvious negative effect on collembolan survival. Over the three generations, the mean $( \pm \mathrm{SE})$ number of juveniles produced per individually fed collembolan was $22.57 \pm 0.635$ for 100 samples in the CT set and $20.70 \pm 0.590$ for 99 samples in the BT set. According to Student's $t$-test for each generation, the number of juveniles produced per collembolan did not significantly differ between the CT and BT sets for any of the three generations ( $p=0.912,0.114,0.071$ for generations 1, 2 and 3, respectively) (Fig. 2). Twoway ANOVA (with diet treatment, "generation", and their interaction as fixed factors, Table 1) showed that the mean number of juveniles produced per collembolan was significantly affected by "generation" ( $p=0.001)$ but not by diet treatment $(p=0.068)$ or the interaction between diet treatment and "generation" $(p=0.410)$. The results suggest that even in the same treatment culture condition, the reproductions of $F$. candida were still affected by some unpredictable environment stress instead of the BT diet.

\section{Transcriptome sequencing and annotation.}

A total of 316,693,647 raw reads for 16 samples were generated, and deposited in the NCBI Sequence Read Archive (SRP132745). After data filtering and de novo assembly, 284,174,422 clean reads were assembled into 93,976 unigenes. The total length of these unigenes was $155,829,628 \mathrm{bp}$. The mean length of the unigenes was $1,658 \mathrm{bp}$, and N50 length was 3,292 bp. The sequence data of each type of sample were unbiased, and enough reads were obtained to perform gene expression analyses. The quantity and quality of the RNA sequencing data are shown in Table 2.

Of the total number of unigenes, 55,390 (58.94\%), 17,230 (18.33\%), 47,734 (50.79\%), 43,284 (46.06\%), 31,016 (33.00\%), and 18,603 (19.80\%) were annotated in NCBI Nr, NCBI Nt, SwissProt, KEGG, COG, and GO databases, respectively. Overall, 57,758 (61.46\%) unigenes were annotated to known protein/nucleotide sequences.

\section{Differentially expressed genes (DEGs) over three generations.}

463 DEGs ( $0.49 \%$ of all unigenes) between the CT and BT sets were identified for all three generations (Fig. 3A-C), including 211, 19, and 244 DEGs for the first, second, and third generation, respectively (Fig. 3D). There was no consistent tendency for either the up-regulation or down-regulation of DEGs and there was no consistent co-expression of DEGs over the three generations (Fig. 3E) and only 6 DEGs (5 up-regulated and 1 down-regulated) were identified in both the first and third generations. 5 contra-regulated DEGs were detected in the second and 
318

319

320

321

322

323

324

325

326

327

328

329

330

331

332

333

334

335

336

337

338

339

340

341

342

343

344

345

346

347

348

349

350

351

352

353

354

355

356

357

third generations. The functions of these 11 DEGs are unknown.

The heatmap analysis of DEGs over three generations showed similar expression patterns of the CT and BT sets in each generation (Fig. 4A). The hierarchical clustering of all samples demonstrated that the CT and BT samples of the same generation were always clustered together, instead of the CT or the BT sets of different generations (Fig. 4B).

\section{Biological marker expression profile.}

A total of 855 unigenes for the seven biomarkers (CAT, GST, SOD, GR, CES, MTC, and HSP70) were annotated, but only five unigenes were differentially expressed between the CT and BT sets, i.e., were up- or down-regulated by more than 2-fold in the BT set (Table 3): one unigene of CAT (CL2174.Contig5_All) was up-regulated in the third generation, three unigenes of CES (Unigene7933_All, CL5034.Contig2_All, and CL6365.Contig1_All) encoding carboxylesterase type B were up- or down-regulated in the first or the third generations, one unigene of HSP70 (Unigene15285_All) was greatly down-regulated, i.e., there was a 5-fold decrease in expression, in the first generation. However, the expression of the five DEGs was significantly different in the BT vs. CT sets in only a specific generation, and none of those changes are consistent in all three generations (Table 3). Furthermore, the gene expression hierarchical clustering of the seven markers (Fig. 5) clearly showed that the CT and BT sets from the same generation always clustered together, which confirms that CrylF treatment did not affect the functions related to the seven biological markers.

\section{Discussion}

Multi-generational laboratory tests are useful for detecting the chronic toxicity of pollutants on collembolans (Campiche et al., 2007; van Gestel et al., 2017). Most previous assessments of Bt toxins or crops on collembolans involved the assessment of short-term exposure (one generation) rather than chronic toxicity (Yang et al., 2015, 2018; Zhang et al., 2017a). With the long-term cultivation of $B t$ crops, however, toxicity may be undetectable by the traditional one-generation risk assessment methods and a finite number of physiological indices, which may increase in subsequent generations because of continuous exposure to $B t$ toxins. Therefore, it is necessary to test the effects of $B t$ toxins on non-target organisms with multi-generational toxicological assessments. Two previous laboratory-based multi-generational risk assessments of $B t$ toxins on collembolans (Bakonyi et al., 2011; Szabó, Seres \& Bakonyi, 2017) found that feeding on leaves of $B t$ maize (MON 810) affected egg production, growth rate, and food preference in F. candida. In addition, Yuan et al. (2011) found that feeding on Bt rice decreased collembolan catalase activity. However, these results are difficult to explain (except to note that $B t$ and non- $B t$ plant tissues may differ in properties other than the presence or absence of the $B t$ toxin). The current study used a diet that was identical except for the presence or absence of the $B t$ toxin. That $F$. candida is parthenogenetic facilitated the synchronization of generations and the multigenerational assessment. We did not use a positive control, because most positive controls such 
as insecticides usually seriously affect the survival and the development of $F$. candida, making it hard to carry out multi-generational exposure experiments. It is challenging to find an appropriate positive control in a multi-generational study, to appropriately reduce the concentration of the insecticidal compounds, for new individuals for each generation to show exposure, or to find another species that can survive multiple generations with sub-lethal effects.

In addition to comparing $F$. candida survival and reproduction, the current study also considered the effects of the $B t$ toxin on gene expression. Microarray-based detection methods have been widely used to evaluate the molecular effect of pollutants on test organisms (Baillon et al., 2016; Roelofs et al., 2009; Yuan et al., 2014). Compared with microarray, RNA-Seq is more comprehensive and is better able to detect a potential risk, since it does not rely on a predesigned complement sequence detection probe it enables the identification of genetic variants and it can quantify and profile overall gene expression, including rare and novel transcripts (Montgomery et al., 2010; Sultan et al., 2008; Wang, Gerstein \& Snyder, 2009). If the pollutants are harmful to $F$. candida, they should create a constant stress and result in the co-expression of DEGs at different exposure times (Qiao et al., 2015) but this was not the case in the current study. The expression pattern of DEGs was more similar between the CT and BT sets in the same generation than between the same diet treatment of different generations (Fig. 4) suggesting that the detected DEGs may be random events perhaps caused by uncontrolled conditions in the experiment rather than by Cry1F. Moreover, the statistical analysis showed that the $F$. candida reproduction significantly differed among generations but not between the diet treatments (Table 1), which further confirmed that Cry1F had no constant or cumulative effect on $F$. candida. Although we synchronized $F$. candida to stage before every generation and although we strictly controlled the culture conditions, there were probably some uncontrolled differences in season, diet quality, or other factors between generations.

The biological marker assay is commonly used for monitoring environmental contaminants because it is rapid, simple, and sensitive. Yousef, Abdelfattah \& Augustyniak (2017) found that fertilizer industry pollutants greatly affected the activities of SOD and CAT in the grasshopper Aiolopus thalassinus. By assessing GST responses, Oliveira et al. (2015) assayed the risk of $F$. candida exposure to carbamazepine. Nakamori \& Kaneko (2013) measured the effect of Cd exposure on F. candida by assessing the gene expression of MTC, which is a biomarker related to heavy metal detoxification. Most previous studies only determined the gene expression differences of one or several biomarkers by RT-qPCR. In our study, RNA-Seq provided a whole transcriptome profile and therefore, we were able to screen a large variety of important biomarker genes in F. candida, including some novel response genes.

\section{Conclusions}

In this study, we established a laboratory-based multi-generational risk assessment of Cry1F for $F$. candida. In addition to assessing the effect of Cry $1 F$ on $F$. candida survival and 
398

399

400

401

402

403

404

405

406

407

408

409

410

411

412

413

414

415

416

417

418

419

420

421

422

423

424

425

426

427

428

429

430

431

432

433

434

435

436

437

reproduction, we also assessed the effect on gene expression by using a comparative transcriptome analysis for the first time. Our results demonstrated that Cry1F did not affect the survival or reproduction of $F$. candida over three consecutive generations and did not alter global gene expression levels or the expression profiles of seven sensitive biological markers. In summary, these data indicate that Cry $1 \mathrm{~F}$ does not have harmful effects on the non-target organism $F$. candida.

\section{References}

Baillon, L.; Pierron, F.; Pannetier, P.; Normandeau, E.; Couture, P.; Labadie, P.; Budzinski, H.; Lambert, P.; Bernatchez, L.; Baudrimont, M. Gene transcription profiling in wild and laboratory-exposed eels: Effect of captivity and in situ chronic exposure to pollution. Sci. Total Environ. 2016, 57, 92-102.

Bakonyi, G.; Dolezsai, A.; Mátrai, N.; Székács, A. Effects of consumption of Bt-maize (MON 810) on the collembolan Folsomia candida, over multiple generations: A laboratory study. Insects. 2011, 2(2), 243-252.

Bravo, A.; Likitvivatanavong, S.; Gill, S. S.; Soberón, M. Bacillus thuringiensis: a story of a successful bioinsecticide. Insect Biochem. Mol. Biol. 2011, 41(7), 0-431.

Buch, A. C.; Niemeyer, J.C.; Correia, M. E. F.; Silva, E. V. Ecotoxicity of mercury to Folsomia candida and Proisotoma minuta (Collembola: Isotomidae) in tropical soils: Baseline for ecological risk assessment. Ecotox. Environ. Safe. 2016, 127, 22-29.

Campiche, S.; L'Arnbert, G.; Tarradellas, J.; Becker-van Slooten, K. Multigeneration effects of insect growth regulators on the springtail Folsomia candida. Ecotox. Environ. Safe. 2007, 67(2), 180-189.

Chen, G.; den Braver, M. W.; van Gestel, C. A. M.; van Straalen, N. M.; Roelofs, D. Ecotoxicogenomic assessment of diclofenac toxicity in soil. Environ. Pollut. 2015, 199, 253-260.

Clark, B. W.; Phillips, T.A.; Coats, J. R. Environmental fate and effects of Bacillus thuringiensis (Bt) proteins from transgenic crops: A review. J. Agr. Food Chem. 2005, 53(12), 4643-4653.

European Food Safety Authority [EFSA]. Guidance on the environmental risk assessment of genetically modified plants. EFSA J. 2010.

Gao, Y. J.; Zhu, H. J.; Chen, Y.; Li, Y. H.; Peng, Y. F.; Chen, X. P. Safety Assessment of Bacillus thuringiensis insecticidal proteins Cry1C and Cry2A with a zebrafish embryotoxicity test. J. Agr. Food Chem. 2018, 66(17), 4336-4344.

van Gestel, C, A, M,; Silva, C. D. L. E.; Lam, T.; Koekkoek, J. C.; Lamoree, M. H.; Verweij, R. A. Multigeneration toxicity of imidacloprid and thiacloprid to Folsomia candida. Ecotoxicology. 2017, 26(3), 1-9.

Higgins, L. S.; Babcock, J.; Neese, P.; Layton, R. J.; Moellenbeck, D. J.; Storer, N. Three-year field monitoring of Cry1F, event DAS-Ø15Ø7-1, maize hybrids for nontarget arthropod effects. Environ. Entomol. 2009, 38(1), 281-292. 
Hopkin, S. P. Biology of the springtails (Insecta: Collembola). Oxford University Press, NY, 1997.

Icoz, I.; Stotzky, G. Fate and effects of insect-resistant Bt crops in soil ecosystems. Soil Biol. Biochem. 2008, 40, 559-586.

ILSI (International Life Sciences Institute) Research Foundation. A Review of the Environmental Safety of the Cry1F Proteins. ILSI Research Foundation, Washington DC. 2013. http://ilsirf.org/publication/a-review-of-the-environmental-safety-of-the-crylfprotein/

International Service for the Acquisition of Agri-biotech Applications [ISAAA]. Global Status of Commercialized Biotech/GM Crops: 2016. ISAAA Brief No. 52. 2016. ISAAA: Ithaca, New York, USA.

Jia, H. R.; Geng, L. L.; Li, Y. H.; Wang, Q.; Diao, Q. Y.; Zhou, T.; Dai, P. L. The effects of Bt Crylle toxin on bacterial diversity in the midgut of Apis mellifera ligustica (Hymenoptera: Apidae). Sci. Rep. 2016, 6, 24664.

Jiao, Y. Y.; Yang, Y.; Meissle, M.; Peng, Y. F.; Li, Y. H. Comparison of susceptibility of Chilo suppressalis and Bombyx mori to five Bacillus thuringiensis proteins. J. Invertebr. Pathol. 2016, 136, 95-99.

Kim, Y. H.; Hwang, C. E.; Kim, T. S.; Lee, J. H.; Lee, S. H. Assessment of potential impacts due to unintentionally released $\mathrm{Bt}$ maize plants on non-target aphid Rhopalosiphum padi (Hemiptera: Aphididae). J. Asia-Pac. Entomol. 2012, 15(3), 443-446.

Li, Y. H.; Romeis, J.; Wu, K. M.; Peng, Y. F. Tier-1 assays for assessing the toxicity of insecticidal proteins produced by genetically engineered plants to non-target arthropods. Insect Sci. 2014, 21(2), 125-134.

Li, Y. H.; Wu, K. M.; Zhang, Y. J.; Yuan, G. H. Degradation of Cry1 Ac protein within transgenic Bacillus thuringiensis rice tissues under field and laboratory conditions. Environ. Entomol. 2007, 36(5), 1275-1282.

Liu, Q. S.; Hallerman, E.; Peng, Y. F.; Li, Y. H. Development of Bt rice and $B t$ maize in China and their efficacy in target pest control. Int. J. Mol. Sci. 2016, 17(10), 1561.

Liu, Y. R.; Zheng, Y. M.; Zhang, L. M.; Luan, Y. X.; He, J. Z. Effects of mercury on reproduction, avoidance, and heat shock protein gene expression of the soil springtail Folsomia candida. Environ. Toxicol. Chem. 2010, 30(7), 654-659.

Marques, L. H.; Santos, A. C.; Castro, B. A.; Storer, N. P.; Babcock, J. M.; Lepping, M. D.; Sa, V.; Moscardini, V. F.; Rule, D. M.; Fernandes, O. A. Impact of transgenic soybean expressing Cry $1 \mathrm{Ac}$ and Cry $1 \mathrm{~F}$ proteins on the non-target arthropod community associated with soybean in Brazil. Plos One. 2018, 13(2), e0191567.

Montgomery, S. B.; Sammeth, M.; Gutierrez-Arcelus, M.; Lach, R. P.; Ingle, C.; Nisbett, J.; Guigo, R.; Dermitzakis, E. T. Transcriptome genetics using second generation sequencing in a Caucasian population. Nature. 2010, 464(7289), 773-777.

Nakamori, T.; Kaneko, N. Biomarker responses reveal that food quality affects cadmium exposure in the soil collembolan Folsomia candida. Environ. Pollut. 2013, 176, 165-170. Neher, D. A.; Muthumbi, A. W. N.; Dively G. 2014. Impact of coleopteran-active Bt corn on 
non-target nematode communities in soil and decomposing corn roots. Soil. Biol. Biochem. 2014, 76, 127-135.

Noordhoek, J. W.; Jeroen, N.; Fabrizia, P.; Ida, B.; Oscar, F.; Kora, M. W.; Janine, M.; Rudo, A. V.; Cornelis, A. M. G.; Nico, M. S.; Dick, R. Phenotypic and transcriptional responses associated with multi-generation exposure of Folsomia candida to engineered nanomaterials. Environ. Sci.: Nano. 2018, 5, 2426-2439.

Niu, L.; Ma, W. H.; Lei, C. L.; Jurat-Fuentes, J. L.; Chen, L. Z. Herbicide and insect resistant Bt cotton pollen assessment finds no detrimental effects on adult honey bees. Environ. Pollut. 2017, 230, 479-485.

Organisation for Economic Cooperation and Development [OECD]. Test No. 232: Collembolan Reproduction Test in Soil. OECD Publishing, Paris, France, 2016.

Oliveira, M.; Cardoso, D. N.; Soares, A. M. V. M.; Loureiro, S. Effects of short-term exposure to fluoxetine and carbamazepine to the collembolan Folsomia candida. Chemosphere. 2015, $120,86-91$.

Qiao, M.; Wang, G. P.; Zhang, C.; Roelofs, D.; van Straalen, N. M.; Zhu, Y. G. Transcriptional profiling of the soil invertebrate Folsomia candida in pentachlorophenol-contaminated soil. Environ. Toxicol. Chem. 2015, 34(6), 1362-1368.

Roelofs, D.; Janssens, T. K. S.; Timmermans, M. J. T. N.; Nota, B.; Mariën, J.; Bochdanovits, Z.; Ylstra, B.; van Straalen, N. M. Adaptive differences in gene expression associated with heavy metal tolerance in the soil arthropod Orchesella cincta. Mol. Ecol. 2009, 18(15), $3227-3239$.

Romeis, J.; Bartsch, D.; Bigler, F.; Candolfi, M. P.; Gielkens, M. M. C.; Hartley, S. E.; Hellmich, R. L.; Huesing, J. E.; Jepson, P. C.; Layton, R.; Quemada, H.; Raybould, A.; Rose, R. I.; Schiemann, J., Sears, M. K.; Shelton, A. M.; Sweet, J.; Vaituzis, Z.; Wolt, J. D. Assessment of risk of insect-resistant transgenic crops to nontarget arthropods. Nat. Biotechnol. 2008, 26(2), 203-208.

Shelton, A. M.; Zhao, J. Z.; Roush, R. T. Economic, ecological, food safety, and social consequences of the deployment of Bt transgenic plants. Annu. Rev. Entomol. 2002, 47, $845-881$.

Sims, S. R.; Martin, J. W. Effect of the Bacillus thuringiensis insecticidal proteins CryIA(b), CryIA(c), CryIIA, and CryIIIA on Folsomia candida and Xenylla grisea (Insecta: Collembola). Pedobiologia. 1997, 41(5), 412-416.

Sultan, M.; Schulz, M. H.; Richard, H.; Magen, A.; Klingenhoff, A.; Scherf, M.; Seifert, M.; Borodina, T.; Soldatov, A.; Parkhomchuk, D.; Schmidt, D.; O’Keeffe, S.; Haas, S.; Vingron, M.; Lehrach, H.; Yaspo, M. L. A global view of gene activity and alternative splicing by deep sequencing of the human transcriptome. Science. 2008, 321(5891), 956-960.

Svobodová, Z.; Skoková, H. O.; Hutchison, W. D.; Hussein, H. M.; Sehnal, F. Risk assessment of genetically engineered maize resistant to Diabrotica spp.: influence on above-ground arthropods in the Czech Republic. Plos One. 2015, 10(6), e0130656.

Szabó, B.; Seres, A.; Bakonyi, G. Long-term consumption and food replacement of near-isogenic 
by Bt-maize alter life-history traits of Folsomia candida Willem 1902 (Collembola). Appl. Ecol. Env. Res. 2017, 15(4), 1275-1286.

Tian, J. C.; Wang, X. P.; Long, L. P.; Romeis, J.; Naranjo, S. E.; Hellmich, R. L.; Shelton, A. M. Eliminating host-mediated effects demonstrates Bt maize producing Cry1F has no adverse effects on the parasitoid Cotesia marginiventris. Transgenic Res. 2014, 23(2), 257-264.

Valldor, P.; Miethling-Graff, R.; Martens, R.; Tebbe, C. C. Fate of the insecticidal Cry1 Ab protein of GM crops in two agricultural soils as revealed by ${ }^{14} \mathrm{C}$-tracer studies. Appl. Microbiol. Biot. 2015, 99(17), 7333-7341.

Wang, Z.; Gerstein, M.; Snyder, M. RNA-Seq: a revolutionary tool for transcriptomics. Nat. Rev. Genet. 2009, 10(1), 57-63.

Yang, Y.; Chen, X.P.; Cheng, L. S.; Cao, F. Q.; Romeis, J.; Li, Y. H.; Peng, Y. F. Toxicological and biochemical analyses demonstrate no toxic effect of Cry1C and Cry2A to Folsomia candida. Sci. Rep. 2015, 5, 15619.

Yang, Y.; Zhang, B.; Zhou, X.; Romeis, J.; Peng, Y. F.; Li, Y. H. Toxicological and biochemical analyses demonstrate the absence of lethal or sublethal effects of crylC- or cry $2 A$ expressing Bt rice on the collembolan Folsomia candida. Front. Plant Sci. 2018, 9, 131.

Yousef, H. A.; Abdelfattah, E. A.; Augustyniak, M. Evaluation of oxidative stress biomarkers in Aiolopus thalassinus (Orthoptera: Acrididae) collected from areas polluted by the fertilizer industry. Ecotoxicology. 2017, 26(3), 340-350.

Yuan, Y. Y.; Ke, X.; Chen, F. J.; Krogh, P. H.; Ge, F. Decrease in catalase activity of Folsomia candida fed a Bt rice diet. Environ. Pollut. 2011, 159(12), 3714-3720.

Yuan, Y. Y.; Krogh, P. H.; Bai, X.; Roelofs, D.; Chen, F. J.; Zhu-Salzman, K.; Liang, Y. Y.; Sun, Y. C.; Ge, F. Microarray detection and qPCR screening of potential biomarkers of Folsomia candida (Collembola: Isotomidae) exposed to Bt proteins (Cry1 Ab and Cry1 Ac). Environ. Pollut. 2014, 184, 170-178.

Zhang, B.; Yang, Y.; Zhou, X.; Shen, P.; Peng, Y. F.; Li, Y. H. A laboratory assessment of the potential effect of Cry1 Ab/Cry2Aj-containing Bt maize pollen on Folsomia candida by toxicological and biochemical analyses. Environ. Pollut. 2017a, 222, 94-100.

Zhang, X. H.; Ruan, Z. Q.; You, X. X.; Wang, J. T.; Chen, J. M.; Peng, C.; Shi, Q. De novo assembly and comparative transcriptome analysis of the foot from Chinese green mussel (Perna viridis) in response to cadmium stimulation. Plos One. 2017b, 12(5), e0176677.

Zhao, Z.; Li, Y.; Xiao, Y.; Abid, A.; Hussain, D. K.; Chen, W.; Wu, K. Distribution and metabolism of Bt-Cry1 Ac toxin in tissues and organs of the cotton bollworm, Helicoverpa armigera. Toxins. 2016, 8(7), 212.

Zortéa, T.; Baretta, D.; Maccari, A. P.; Segat, J. C.; Boiago, E. S.; Sousa, J. P.; Da Silva, A. S. Influence of cypermethrin on avoidance behavior, survival and reproduction of Folsomia candida in soil. Chemosphere. 2015, 122, 94-98. 


\section{Table $\mathbf{1}$ (on next page)}

A two-way ANOVA of reproduction ${ }^{a}$ with diet treatment, "generation", and their interaction as fixed factors, followed by LSD test. 
1 Table 1. A two-way ANOVA of reproduction ${ }^{a}$ with diet treatment, "generation" , and their

2 interaction as fixed factors, followed by LSD test.

\begin{tabular}{lccccc}
\hline source & $\mathrm{SS}$ & $d f$ & $\mathrm{MS}$ & $F$ & $p$-value \\
\hline diet treatment & 0.052 & 1 & 0.052 & 3.363 & 0.068 \\
"generation" & 0.227 & 2 & 0.114 & 7.414 & $0.001^{*}$ \\
$\begin{array}{c}\text { diet treatment } \\
\quad\end{array}$ & 0.027 & 2 & 0.014 & 0.896 & 0.410 \\
"generation" & & & & & \\
error & 2.956 & 193 & 0.015 & & \\
total & 348.507 & 199 & & & \\
\hline
\end{tabular}

$3{ }^{a}$ The number of juveniles produced per collembolan in the individual feeding test was calculated 4 as reproduction value.

$5 b$ "generation" is a label representing unknow variable factors, in order to test whether the 6 reproductions of $F$. candida over three generations in the same culture condition were still 7 affected by unpredictable environment stress.

$8 * p<0.05$. 


\section{Table 2 (on next page)}

Summary of $F$. candida transcriptome sequencing and assembly for $\mathrm{CT}$ and $\mathrm{BT}$ sets over three generations. 
1 Table 2. Summary of $F$. candida transcriptome sequencing and assembly for CT and BT sets

2 over three generations.

3

\begin{tabular}{lcccccc}
\hline \multirow{2}{*}{ statistic } & \multicolumn{3}{c}{ CT } & \multicolumn{4}{c}{ BT } \\
\cline { 2 - 7 } & 1st & 2nd & 3rd & 1st & 2nd & 3rd \\
\hline raw reads & $55,491,201$ & $52,314,588$ & $51,127,722$ & $55,080,974$ & $49,522,385$ & $53,156,777$ \\
clean reads & $51,147,377$ & $43,971,149$ & $47,089,957$ & $50,778,618$ & $42,728,374$ & $48,458,947$ \\
Q20 (\%) & 98.21 & 98.46 & 99.00 & 98.33 & 98.36 & 98.80 \\
GC content (\%) & 40.63 & 42.93 & 42.96 & 41.97 & 43.63 & 43.84 \\
\hline
\end{tabular}

4

5 


\section{Table 3 (on next page)}

Summary of annotated unigenes and DEGs ${ }^{a}$ of seven biomarkers of $F$. candida over three generations. 
1 Table 3. Summary of annotated unigenes and DEGs ${ }^{a}$ of seven biomarkers of $F$. candida over

2 three generations.

3

\begin{tabular}{|c|c|c|c|c|c|c|c|c|}
\hline \multirow{2}{*}{ biomarker } & \multirow{2}{*}{$\begin{array}{l}\text { number of } \\
\text { unigenes }\end{array}$} & \multirow{2}{*}{ name of unigene } & \multicolumn{2}{|c|}{$1 \mathrm{st}$} & \multicolumn{2}{|c|}{$2 \mathrm{nd}$} & \multicolumn{2}{|c|}{$3 \mathrm{rd}$} \\
\hline & & & $\mathrm{FC}^{b}$ & FDR & $\mathrm{FC}$ & FDR & $\mathrm{FC}$ & FDR \\
\hline CAT & 52 & CL2174.Contig5_All & 0.443 & 1.000 & 1.859 & 1.000 & 2.857 & $0.042 *$ \\
\hline GST & 191 & - & - & - & - & - & - & - \\
\hline SOD & 42 & - & - & - & - & - & - & - \\
\hline GR & 6 & - & - & - & - & - & - & - \\
\hline \multirow{3}{*}{ CES } & \multirow{3}{*}{408} & Unigene7933_All & 1.664 & $0.042 *$ & -2.469 & 1.000 & 0.225 & 1.000 \\
\hline & & CL5034.Contig2_All & -1.968 & $0.023^{*}$ & 0.366 & 1.000 & -0.410 & 1.000 \\
\hline & & CL6365.Contig1_All & 0.794 & 1.000 & -0.104 & 1.000 & 2.054 & $0.000 *$ \\
\hline MTC & 38 & - & - & - & - & - & - & - \\
\hline HSP70 & 118 & Unigene15285_All & -5.226 & $0.043 *$ & -2.191 & 1.000 & -2.341 & 1.000 \\
\hline SUM & 855 & & & & & & & \\
\hline
\end{tabular}

$4 \quad{ }^{a}$ DEGs: $|\mathrm{FC}| \geq 1, \mathrm{FDR} \leq 0.05$.

$5 \quad{ }^{b} \mathrm{FC}: \log 2$ ratio of FPKM (BT vs. CT).

$6 * \mathrm{FDR} \leq 0.05$

7 DEGs $=$ differentially expressed genes; $\mathrm{FC}=$ fold change; FDR $=$ false discovery rate; FPKM = 8 fragments per kb per million fragments; CAT = catalase; GST = glutathione S-transferase; $\mathrm{SOD}=$ 9 superoxide dismutase; $\mathrm{GR}=$ glutathione reductase; $\mathrm{CES}=$ carboxylesterase; $\mathrm{MTC}=$ 10 metallothionein-like motif containing protein; HSP70 = heat shock protein 70. 


\section{Figure 1}

Culture of Folsomia candida and the flow chart of the experiment.

(A) F. candida reared in a Petri dish with baker's yeast as food. (B) A living individual of $F$. candida. (C) A cluster of $F$. candida eggs. (D) The flow chart of the dietary exposure experiment over three consecutive generations. The CT set of collembolans was fed yeast powder without Cry1F, and the BT set was fed yeast powder with Cry1F. In each generation, both CT and BT sets were fed and evaluated individually (with 30 or 40 replicates) or in groups of 60 to 80 individuals (with two or three replicates).

A

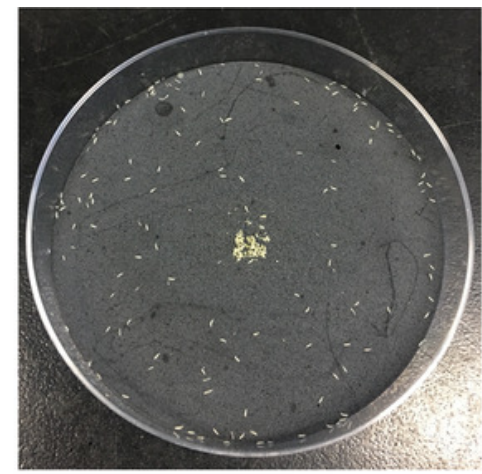

D

\begin{tabular}{|c|c|c|c|c|c|c|c|c|c|}
\hline Generation & \multicolumn{3}{|c|}{$1 \mathrm{st}$} & \multicolumn{3}{|c|}{2 nd } & \multicolumn{3}{|c|}{$3 \mathrm{rd}$} \\
\hline \multirow{5}{*}{ DEE } & \multicolumn{2}{|c|}{ Synchronization } & & \multicolumn{2}{|c|}{ Synchronization } & & \multicolumn{2}{|c|}{ Synchronization } & \\
\hline & & 10 days & & & 10 days & & & \multirow{3}{*}{\multicolumn{2}{|c|}{ 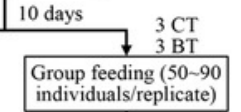 }} \\
\hline & \multirow{2}{*}{$\begin{array}{l}30 \mathrm{BT} \\
\text { Individual feeding (1) } \\
\text { individual/replicate) }\end{array}$} & & $3 \mathrm{BT}$ & \multirow{2}{*}{\begin{tabular}{|l|}
$40 \mathrm{ATT}$ \\
\begin{tabular}{|l|} 
Individual feeding (1) \\
individual/replicate)
\end{tabular}
\end{tabular}} & & $2 \mathrm{BT}$ & \multirow{2}{*}{$\begin{array}{l}40 \mathrm{CT} \\
\begin{array}{l}\text { Individual feeding (1) } \\
\text { individual/replicate) }\end{array}\end{array}$} & & \\
\hline & & \begin{tabular}{|l} 
Group \\
indivic
\end{tabular} & $\begin{array}{l}\text { ing }(50-90-90 \\
\text { replicate) }\end{array}$ & & $\begin{array}{l}\text { Group f } \\
\text { individ }\end{array}$ & $\begin{array}{l}\text { ing }(50 \sim 90 \\
\text { replicate })\end{array}$ & & & \\
\hline & 25 days & & 25 days & \begin{tabular}{l|l}
25 days
\end{tabular} & & 25 days & 25 days & & 25 days \\
\hline & & ᄃ & $\downarrow$ & & $\sqrt{5}$ & $\downarrow$ & & 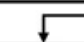 & 7 \\
\hline Test & $\begin{array}{l}\text { Survival and } \\
\text { reproduction } \\
\text { count }\end{array}$ & ELISA & RNA-seq & $\begin{array}{l}\text { Survival and } \\
\text { reproducation } \\
\text { cuunt }\end{array}$ & ELISA & RNA-seq & $\begin{array}{l}\text { Survival and } \\
\text { reproduction } \\
\text { count }\end{array}$ & ELISA & RNA-seq \\
\hline
\end{tabular}




\section{Figure 2}

The reproduction (number of juveniles produced per individually fed collembolan ) of three generations of $F$. candida as affected by addition of CrylF to an artificial diet.

The collembolans were individually fed pure yeast powder (CT set) or yeast powder + Cry1F protein (BT set). Values are means \pm SE $(n=24-38)$. Across all generations, independent Student's t-test between CT and BT sets for each generation indicate no significant difference, with $p=0.912,0.114,0.071$ for the 1 st, 2 nd and 3 rd generations, respectively (significant difference $p<0.05$ ).

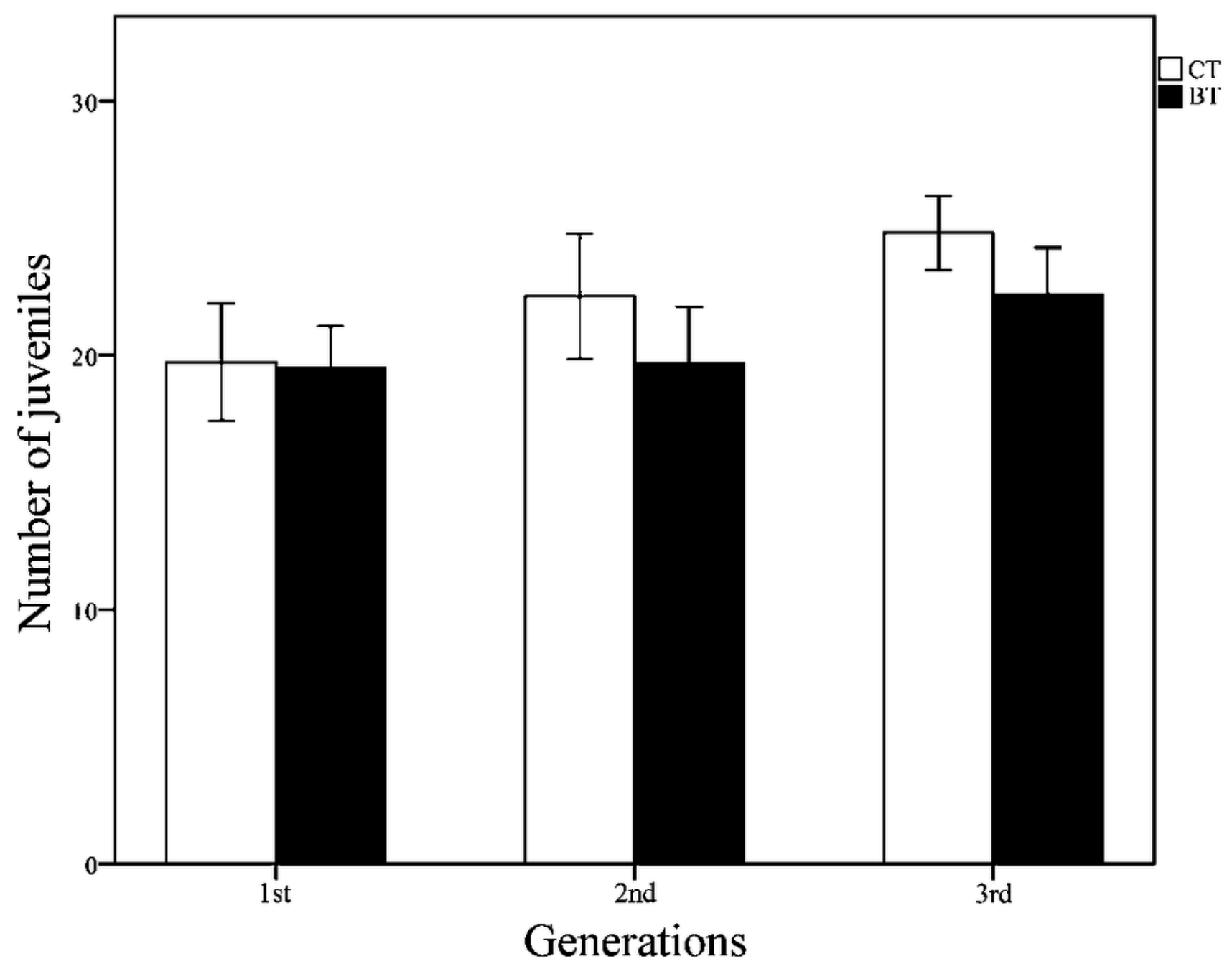




\section{Figure 3}

DEGs in F. candida fed diets with and without CrylF over three generations.

(A-C) Volcano plots of DEGs for the 1st, 2nd and 3rd generation, respectively. DEGs: FC (|log2 ratio| of FPKM (BT vs. CT)) $\geq 1, F D R \leq 0.05$. (D) Numbers of up-regulated and down-regulated DEGs. (E) Venn diagrams of DEGs in three generations. DEGs = differentially expressed genes; $F C=$ fold change; FPKM $=$ fragments per kb per million fragments; FDR $=$ false discovery rate.
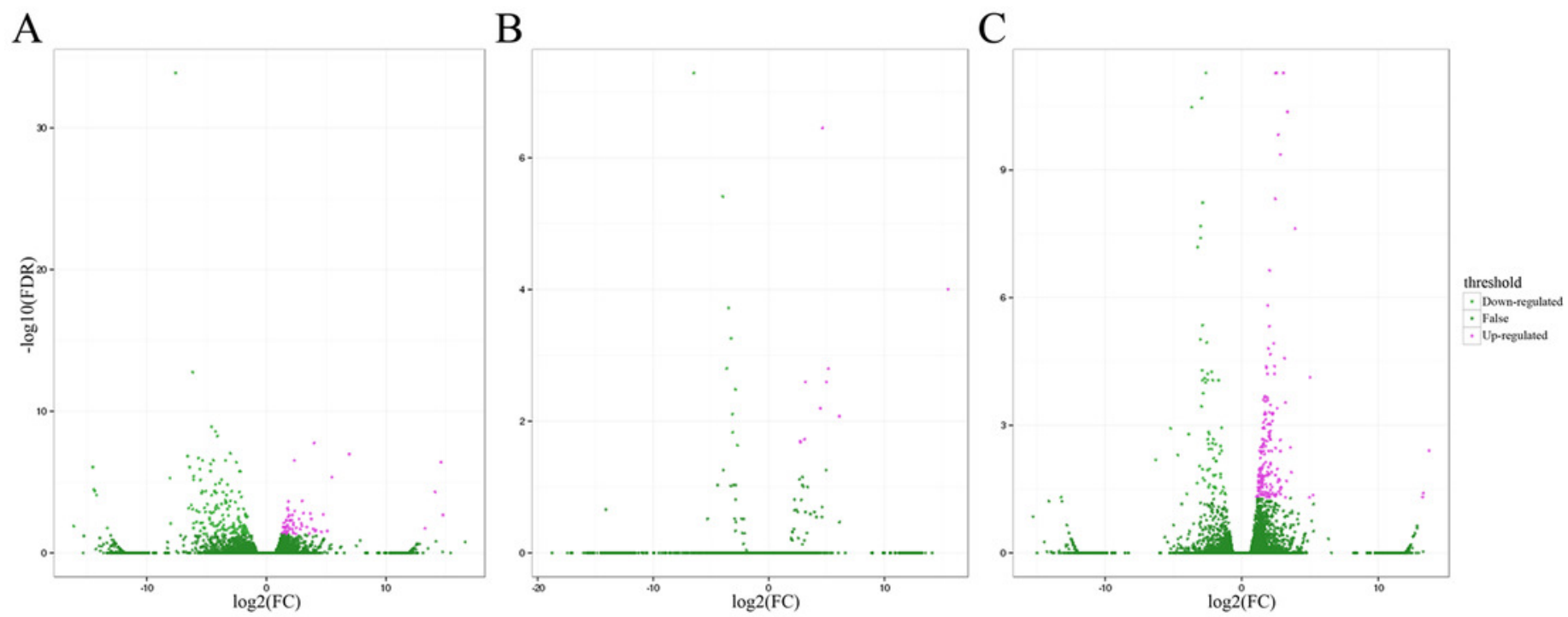

$\mathrm{D}$

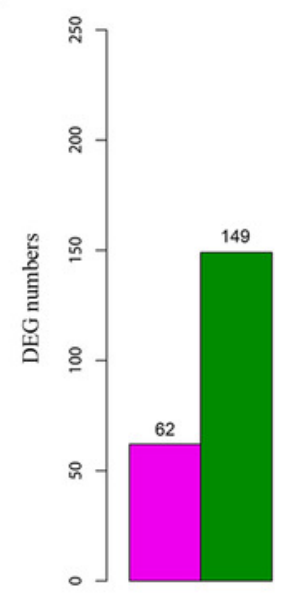

Ist

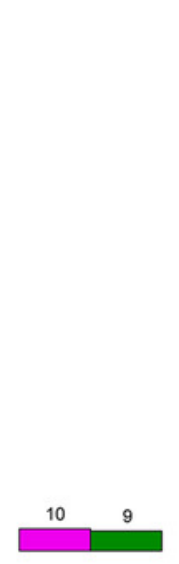

2nd Generations

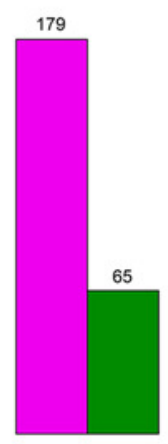

$3 r d$
E

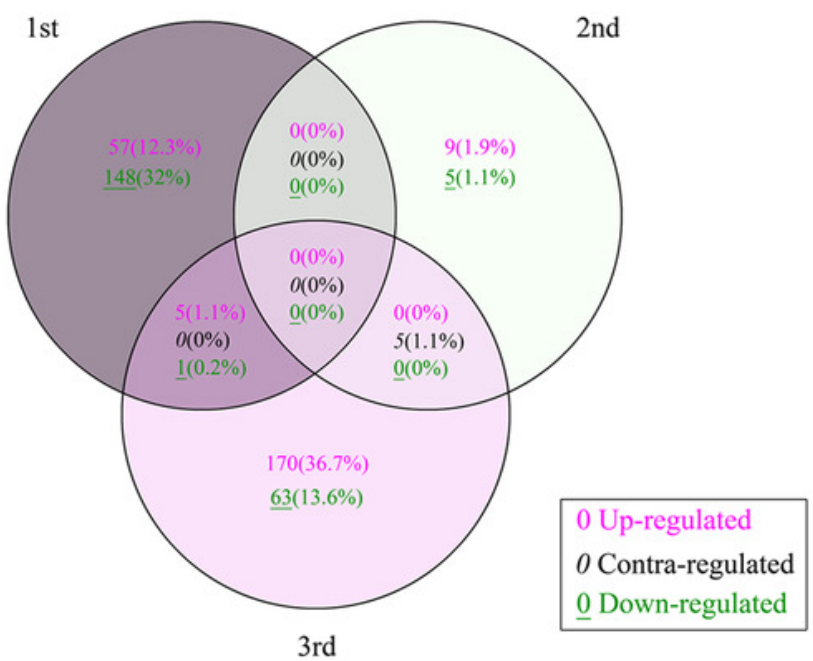




\section{Figure 4}

Heatmap and hierarchical clustering dendrogram of DEGs.

(A) Heatmap of DEGs. Gene clusters were subjected to Gene Ontology analysis. Columns represent samples. The scale bar indicates log-transformed ( common logarithm of the counts plus 1 ) gene expression values, with high expression depicted in red and low expression in green. (B) Hierarchical clustering dendrogram of six samples over three generation. Log-transformed data of DEGs were used for analysis. The distance method was set as 'euclidean' and the cluster method was set as 'complete'.

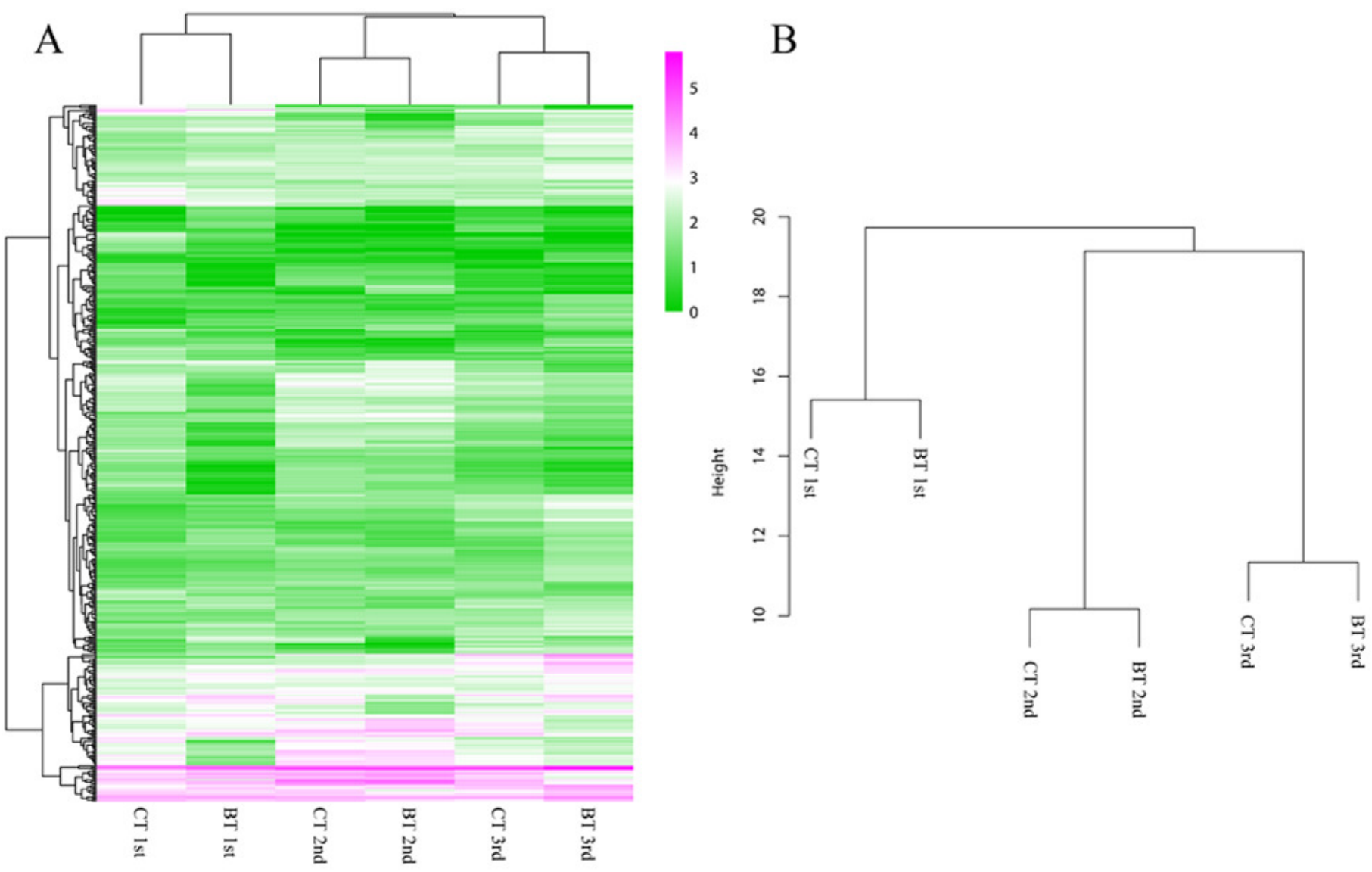




\section{Figure 5}

Heatmap of seven biomarkers.

Gene clusters from hierarchical classification were subjected to Gene Ontology analysis.

Columns represent samples. The scale bar indicates log-transformed gene expression values, with high expression depicted in red and low expression in green. (A) Carboxylesterase (CES). (B) Glutathione S-transferase (GST). (C) Heat shock protein 70 (HSP70). (D) Catalase (CAT). (E) Glutathione reductase (GR). (F) Superoxide dismutase (SOD). (G) Metallothioneinlike motif containing protein (MTC).

A

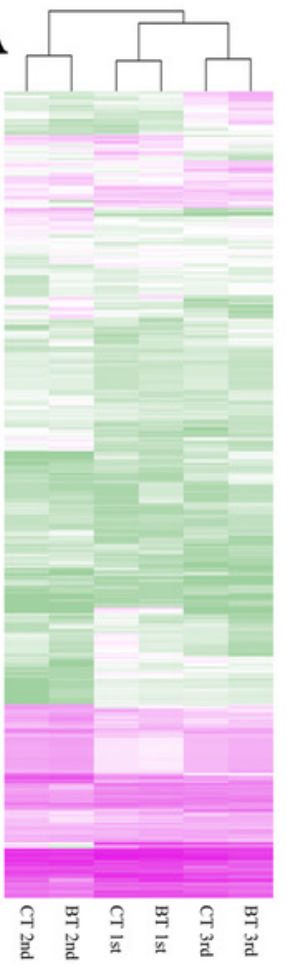

B

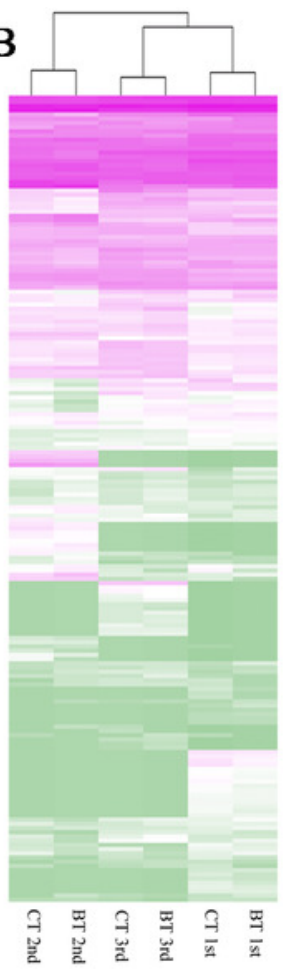

C

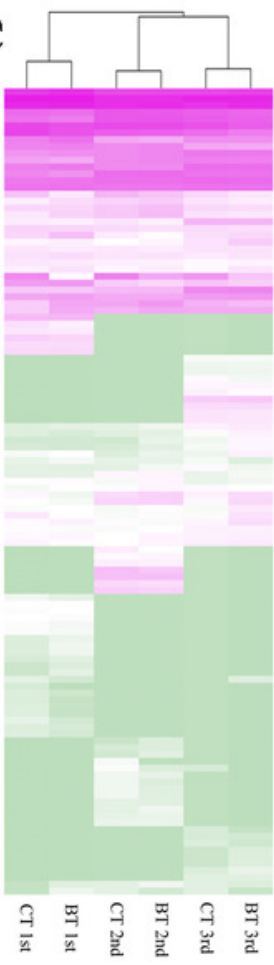

D

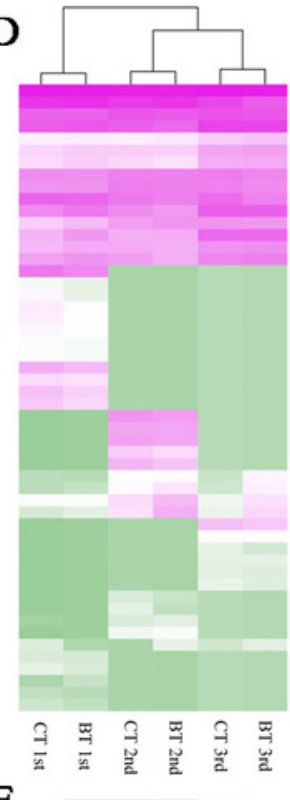

E

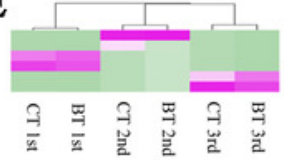

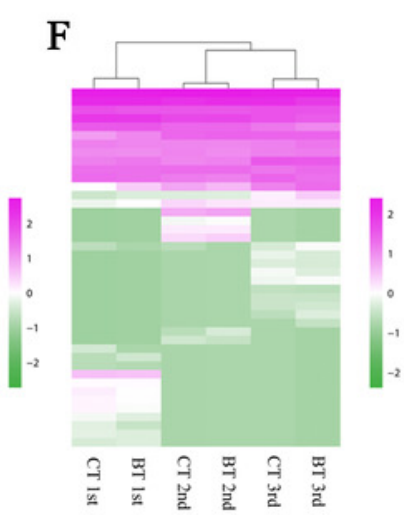

\section{G}

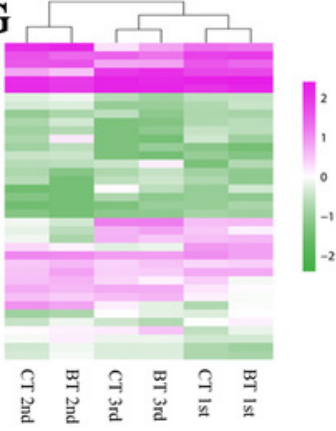

